\title{
Distributed Intrusion Monitoring System With Fiber Link Backup and On-Line Fault Diagnosis Functions
}

\author{
Jiwei XU, Huijuan WU*, and Shunkun XIAO \\ Key Laboratory of Optical Fiber Sensing \& Communications (Ministry of Education), University of Electronic Science \\ and Technology of China, Chengdu, 611731, China \\ ${ }^{*}$ Corresponding author: Huijuan WUＥ-mail: hjwu@uestc.edu.cn
}

\begin{abstract}
A novel multi-channel distributed optical fiber intrusion monitoring system with smart fiber link backup and on-line fault diagnosis functions was proposed. A $1 \times N$ optical switch was intelligently controlled by a peripheral interface controller (PIC) to expand the fiber link from one channel to several ones to lower the cost of the long or ultra-long distance intrusion monitoring system and also to strengthen the intelligent monitoring link backup function. At the same time, a sliding window auto-correlation method was presented to identify and locate the broken or fault point of the cable. The experimental results showed that the proposed multi-channel system performed well especially whenever any a broken cable was detected. It could locate the broken or fault point by itself accurately and switch to its backup sensing link immediately to ensure the security system to operate stably without a minute idling. And it was successfully applied in a field test for security monitoring of the 220-km-length national borderline in China.
\end{abstract}

Keywords: Multi-channel, distributed optical fiber sensing system, cable fault diagnosis, auto-correlation

Citation: Jiwei XU, Huijuan WU, and Shunkun XIAO, "Distributed Intrusion Monitoring System With Fiber Link Backup and On-Line Fault Diagnosis Functions," Photonic Sensors, 2014, 4(4): 354-358.

\section{Introduction}

Recently, with the rapid development of the optical fiber sensing (OFS) technology, the OFS system provides a potentially promising solution for many security applications, such as border or perimeter protection, safety monitoring of the oil or gas pipe, large petrochemical plant. Compared with Mach-Zehnder (M-Z), Sagnac interferometers and quasi-distributed fiber Bragg grating (FBG) sensors, the optical time domain reflectometry (OTDR) technique especially demonstrates advantages of the long or ultra-long distance monitoring ability.

The distributed optical fiber sensing system based on optical time domain reflectometry is generally to detect the phase (for phase-sensitive OTDR) or state of polarization (for polarization-sensitive OTDR) change of the backscattering Rayleigh light, to know if there is perturbation and where it is. The basic OTDR sensing theory and application have been extensively explored by Henry F. Taylor, Xiaoyi Bao, and many others [1-6], and many key parameters such as the sensing length, spatial resolution, frequency response range have already been improved up to quite a high level in the world. In the Brillouin-based Omnisens DITEST monitoring system, the remote module is cascaded to extend the sensing length to hundreds of kilometers [7], and by using bi-directional second-order Raman amplification pumped by a long fiber laser formed

Received: 10 August 2014 / Revised version: 8 September 2014

(C) The Author(s) 2014. This article is published with open access at Springerlink.com

DOI: $10.1007 / \mathrm{s} 13320-014-0217-9$

Article type: Regular 
by two FBGs located at both ends of the fiber, the monitor distance of the polarization-sensitive OTDR system can be achieved up to $106 \mathrm{~km}[8]$. However, the added pumps or repeaters always increase the system's configuration complexity, and its single-ended detection convenience is lost. And the system cost is also increased correspondingly. Fiber fault was also explored by Chen-Hung, Woojin Shin et al., and some new techniques were applied to prevent the fiber fault in the passive optical network (PON) [9-10]. For a long distance distributed sensing system, if the fiber cable is broken or cut off, it will result in a failure of a large monitoring area. Thus, on-line cable fault diagnosis in real time in a distributed sensing system is also necessary.

Thus in this paper, a cost effective multi-channel distributed optical fiber intrusion monitoring system is proposed, in which it not only extends the sensing range without any additional pumps or amplification setups, but also increases the smart fiber link backup function to ensure the security system to operate without a minute idling. In contrast to the conventional distributed fiber sensing system, this system can monitor multiple areas simultaneously. In addition, the real-time on-line cable fault detection and location are realized by a sliding window correlation method, which fulfills the intelligence of the distributed intrusion monitoring system.

\section{Multi-channel OTDR monitoring system design and realization}

\subsection{System design and realization}

The configuration of the multi-channel distributed intrusion monitoring system is designed as shown in Fig. 1(a) and realized as in Fig. 1(b). Based on a conventional OTDR system's configuration, the modulated pulse light is gated into the sensing fiber, and the backscattered Rayleigh light is detected by a photo-detector (PD), sampled and processed in PC. And a $1 \times 4$ optical switch is inserted behind the port 2 of a circulator to expand the monitoring route from one to multiple ones. The optical switch is controlled by a microprocessor to cyclically switch to a different fiber link, and the switched route number is sent back to $\mathrm{PC}$ at the same time.

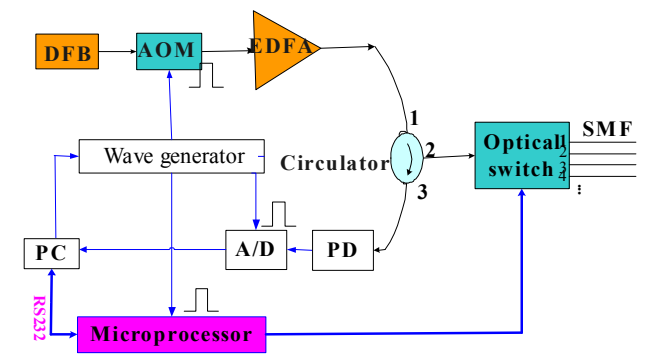

(a) Block diagram

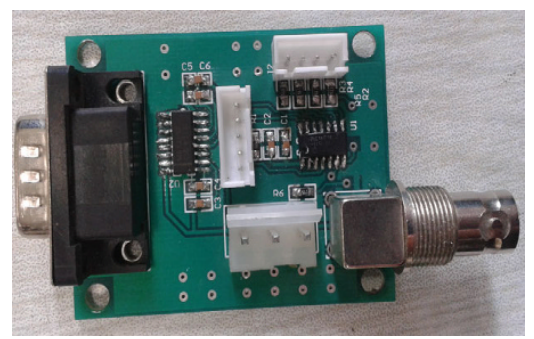

(b) Control board

Fig. 1 Configuration of the multi-channel distributed intrusion monitoring system.

\subsection{Multi-channel's control}

PIC16F1823 is chosen as the microprocessor, and its working principle is shown in Fig. 2. By setting it to work in the pulse capturing mode, when a certain number of the rising (falling) edges of the trigger pulses arrive and are captured by the clock pin (CP), a group of voltage codes from a pre-defined table will be exported via I/O ports, and each code is used to control a certain route switched on and off.

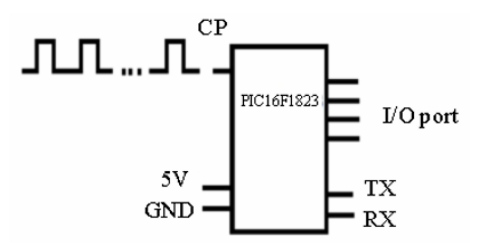

Fig. 2 Working principle of the microprocessor.

\subsection{Channel identification and communication}

When a channel is chosen and switched on, the microprocessor will also send the current channel number to PC through RS232 port. Then, PC can 
identify the current working channel and processes the corresponding data correctly. PC can send other specified commands to the microprocessor through RS232 so that the system can work in other modes intelligently.

\subsection{Backup link is waken up when fiber fault is detected}

In the multi-channel monitoring system as depicted in Fig. 1, the fiber links can be divided into two types: the main working links (channel 1 and channel 3) and the backup links (channel 2 and channel 4). Every adjacent two links are chosen as a group to monitor the same route. Normally, the $1 \times 4$ optical switch only cycles between the main links (channel 1 and channel 3). And the backup link will be woken up while its main link is cut off or broken. Therefore, the system can operate without a minute idling.

\section{Real-time fiber fault diagnosis based on the sliding window auto-correlation mechanism}

\subsection{Principle of weak signal detection based on the auto-correlation characteristics of the signal}

The conventional method to check the situation of the fiber link uses an OTDR, but it has to terminate the monitoring system for checking the cable fault, and has a sluggish response time. Thus, an on-line fiber fault diagnosis method is proposed to detect and locate the cutting or fault points of a cable by using a sliding window auto-correlation method. Figure 3 shows the auto-correlation curves of the Rayleigh scattering signal and the noise without any optical signals, and the data length is 200 samples. We can see that the signal is always

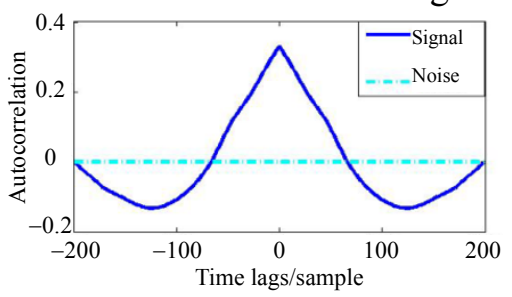

Fig. 3 Auto-correlation curves of the signal and noise. highly correlated with itself but the noise is not. Thus, we can judge if there is an effective signal from its auto-correlation curves.

\subsection{On-line cable fault detection and location using the sliding window auto-correlation mechanism}

The on-line fiber fault diagnosis and location method based on the sliding window auto-correlation mechanism is thus proposed. Assume that the sampled optical time domain signal is $X=\left\{x_{1}, x_{2}, x_{3}, \cdots, x_{l}\right\}$, and auto-correlation is made for each window signal with $M$ samples, as shown in Fig. 4. The window is sliding from end data $x_{l}$ and moving backward to the start $x_{1}$. Calculate the auto-correlation value of each window signal, and set a suitable threshold value which is generally $10^{-4}$. When it is first detected above this level, the current location of the sliding window is just the broken point where the effective optical signal appears. And the fault location is determined by (1):

$$
L=\frac{c}{2 n} \cdot \frac{K_{i}}{f_{s}},\left(1 \leq K_{i} \leq l-M+1\right)
$$

where $c$ is the light speed in vacuum, $n$ is the refractive index of the light in the optical fiber, $f_{s}$ is the sampling frequency, $K_{i}$ is the window index where the cable broken occurs.

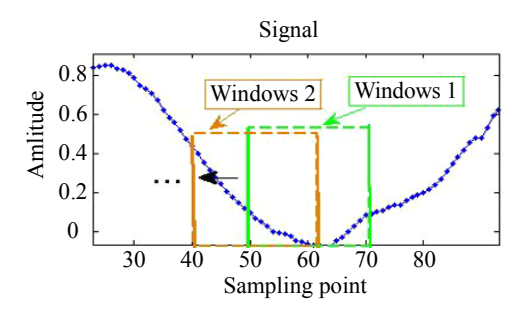

Fig. 4 Sliding windows with a width of 20 samples.

\section{Results and field application}

\subsection{Multi-channel signal acquisition and its fiber cable fault diagnosis results}

In the experiment, a $1 \times 4$ MEMS optical switch was chosen to control two working routes, and a multi-core sensing fiber cable was used for each route. Port 1 and Port 3 of the switch were 
connected to two main links, and Port 2 and Port 4 were connected to their backup links, respectively. And the two routes were about $25 \mathrm{~km}$ and $23 \mathrm{~km}$ long, which were noted as Route 1 and Route 2 . In this multi-channel monitoring system, each working route was switched over every 2 seconds. As shown in Fig. 5, the original OTDR traces of the two routes were acquired every 4 seconds. From the perspective of the client, the two route signals were nearly simultaneously obtained. And we could see that different route data could be accurately separated by this multi-channel system.

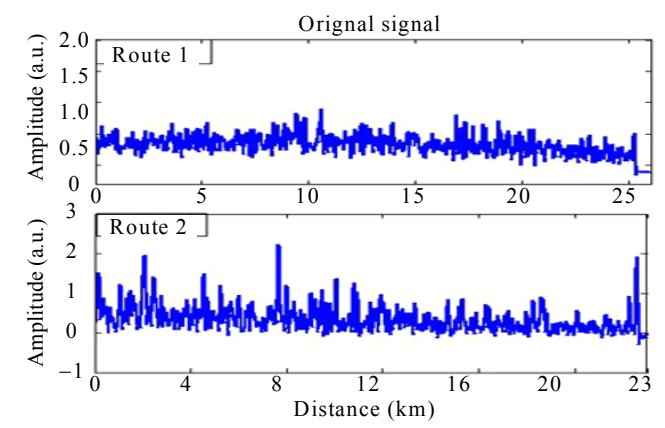

Fig. 5 Original OTDR traces acquired for Route 1 and Route 2.

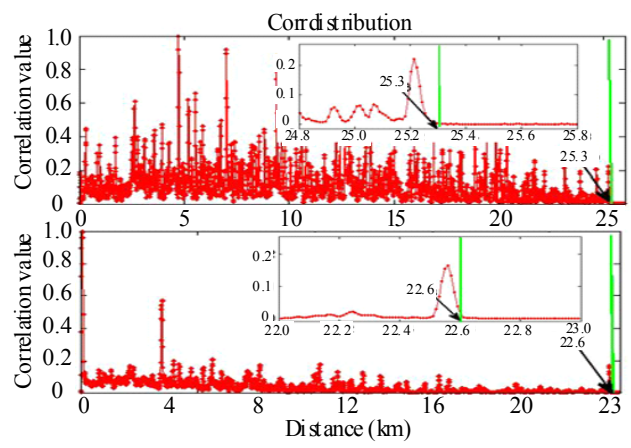

Fig. 6 Spatial correlation curves for Route1 and Route 2.

The synchronous sliding window correlation curves of the two signals are also shown in Fig. 6. It can be seen that the autocorrelation characteristics of the signal and noise (without signal) are clearly distinguished. Thus, the length of the fiber cable can be automatically known. Compared with the amplitude or energy threshold, the correlation threshold has nothing to do with its actual voltage amplitude, and thus it is much easier to set. More fiber fault location results using the real-time autocorrelation method are shown in Table 1. And the traditional OTDR setup was taken as a standard scale in the experiment. It can be seen that the location error of the proposed method is always less than $30 \mathrm{~m}$ and with a much less response time. It is really suitable for this real-time intrusion monitoring system.

Table 1 Fiber fault location results using the real-time autocorrelation method.

\begin{tabular}{cc|ccc}
\hline \multicolumn{2}{c|}{ Standard } & \multicolumn{2}{c}{$\begin{array}{c}\text { Sliding window correlation } \\
\text { method }\end{array}$} & Error \\
\hline OTDR & \multicolumn{2}{c}{ Response } & Detect results & Response time \\
Detect results & time & $25.33 \mathrm{~km}$ & & $\leq 30 \mathrm{~m}$ \\
$25.31 \mathrm{~km}$ & & $13.71 \mathrm{~km}$ & $\leq 2 \mathrm{~s}$ & \\
$13.68 \mathrm{~km}$ & $\geq 30 \mathrm{~s}$ & $5.66 \mathrm{~km}$ & & \\
$5.64 \mathrm{~km}$ & & & \\
\hline
\end{tabular}

\subsection{Borderline security application}

The proposed multi-channel distributed intrusion monitoring system was employed in a 220-km-length national borderline in Xinjiang Province of China. Its configuration, field application photo, and software interface are shown in Figs. 7 and 8. Two phase-sensitive OTDR systems were used for monitoring four routes or four segments of the whole perimeter, and each route ranged from $40 \mathrm{~km}$ to $60 \mathrm{~km}$. By the time-shared control via a $1 \times 4$ optical switch, two routes could be simultaneously monitored by one system's configuration. Thus, longer distance monitoring with lower cost is feasible. In this application, each segment of the borderline had a backup link, which could be awaken when a broken cable was detected and located in order to make sure the monitoring system can operate without a minute idling.
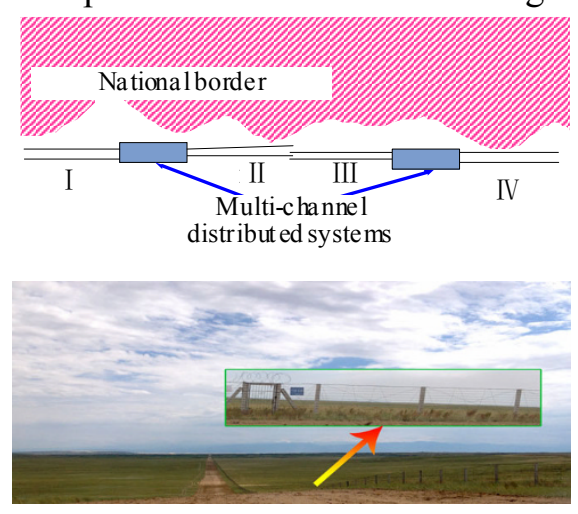

Fig. 7 Field application of the multi-channel distributed intrusion monitoring system in the borderline. 


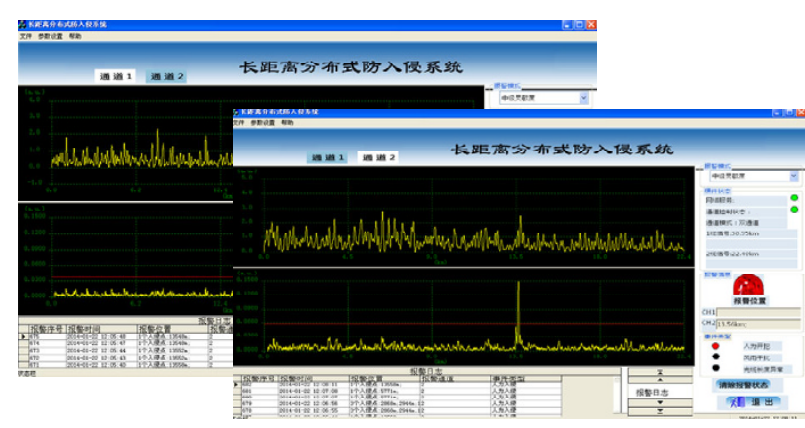

Fig. 8 Software interface of the multi-channel distributed intrusion monitoring system.

\section{Conclusions}

In this paper, an intelligent multi-channel distributed optical fiber intrusion system with the smart fiber link backup and on-line cable fault diagnosis functions is proposed and realized. By using a multi-core fiber cable, the backup link can be woken up when a fault cable is detected and located, which can realize the on-line self-fault-diagnosis, and it can also ensure the monitoring system to operate without a minute idling. The proposed cost effective multi-channel intrusion monitoring system is also suitable for other distributed OTDR systems and can be promising in many significant application areas such as oil/gas pipe lines, electrical power lines, and large-scale civil structures.

\section{Acknowledgment}

The authors gratefully acknowledge the previous supports provided by the National High Technology Research and Development Program of China (863 Program, Grant No. 2007AA01Z245), and the supports provided for this research by the Major Program (Grant No. 61290312) and Youth Foundation (Grant No. 61301275) of the National Science Foundation of China (NSFC), and the Fundamental Research Funds for the Central Universities (Grant No. ZYGX2011J010). This work is also supported by Program for Changjiang Scholars and Innovative Research Team in
University (PCSIRT, IRT1218), and the 111 Project (B14039).

Open Access This article is distributed under the terms of the Creative Commons Attribution License which permits any use, distribution, and reproduction in any medium, provided the original author(s) and source are credited.

\section{References}

[1] J. C. Juarez, E. W. Maier, K. N. Choi, and H. F. Taylor, "Distributed fiber-optic intrusion sensor system," Journal Of Lightwave Technology, 2005, 23(6): 2081-2087.

[2] Z. Y. Zhang and X. Y. Bao, "Distributed optical fiber vibration sensor based on spectrum analysis of Polarization-OTDR system," Optics Express, 2008, 16(14): 10240-10247.

[3] X. Zhang, Y. Lu, F. Wang, H. Liang, and Y. Zhang, "Development of fully-distributed fiber sensors based on Brillouin scattering," Photonic Sensors, 2011, 1(1): 54-61.

[4] Y. Rao, J. Luo, Z. Ran, J. Yue, X. Luo, Z. Zhou, et al., "Long-distance fiber-optic $\Phi$-OTDR intrusion sensing system," in Proc. SPIE, 2009, 7503(1): 75031O-1-75031O-4.

[5] J. Wang, X. Jia, Y. Rao, and H. Wu, "Phase-sensitive optical time-domain reflectometer based on bi-directional Raman amplification," Acta Physica Sinica, 2013, 62(4): 044212-1-044212-5.

[6] Y. J. Rao, "Study on fiber-optic low-coherence interferometric and fiber Bragg grating sensors," Photonic Sensors, 2012, 1(4): 382-400.

[7] M. U. Nikles, H. H. Du, and A. Wang, "Long-distance fiber optic sensing solutions for pipeline leakage, intrusion and ground movement detection," in Proc. SPIE, 2009, 7316(1): 731602-1-731602-13.

[8] F. Peng, Z. Wang, Y. Rao, and X. Jia, "106 km fully-distributed fiber-optic fence based on P-OTDR with 2nd-order Raman amplification," in National Fiber Optic Engineers Conference, Anaheim, California United States, pp. 1-3, 2013.

[9] C. Yeh and S. Chi, "Self-protection against fiber fault for ring-based power-splitting passive optical networks," Optical Engineering, 2008, 47(2): 020501-1-020501-3.

[10] W. Shin, B. A. Yu, Y. L. Lee, T. J. Eom, and Y. C. Noh, "Flexible fiber fault detecting technique based on wavelength swept fiber laser for passive optical network," in Conference on Lasers \& Electro Optics $\&$ The Pacific Rim Conference on Lasers and Electro-Optics, Shanghai, pp. 1-2, 2009. 